\title{
AUDITION AND VISION CHANGES AMONG GERIATRIC RESIDENTS
}

\author{
Kirti Sharma* and Shubha Dube \\ Department of Home Science, University of Rajasthan, Jaipur \\ DOI: http://dx.doi.org/10.24327/ijrsr.2017.0803.0058
}

\begin{tabular}{l}
\hline ARTICLE INFO \\
\hline Article History: \\
Received $15^{\text {th }}$ December, 2016 \\
Received in revised form $25^{\text {th }}$ \\
January, 2017 \\
Accepted $23^{\text {rd }}$ February, 2017 \\
Published online $28^{\text {th }}$ March, 2017 \\
\hline
\end{tabular}

Key Words:

Ageing Adults, Vision, Audition, Quality of life

\begin{abstract}
The process of ageing begins at birth and continues throughout life. Sensory changes can influence the way we see, hear, taste, smell, and respond to touch and pain. All these senses may be affected by ageing, though the dysfunctions of sensations of audition and vision are affected most. This research work explores the levels of vision and hearing related problems among the ageing adults. For the present study the sample comprised of $\mathrm{N}=400$ ageing adults both males and females, in the age range of 65 to $75 y$ years belonging to rural and urban setting of Jaipur district $(n=200$ urban, i.e $100=$ male, $100=$ female and $n=200$ rural i.e. $100=$ male and $100=$ female). Cornell Medical Index Health Questionnaire (Wig et al, 1999) was used to assess ear and eye related problems among the respondents. A very high Myopic condition was prevalent in both the section of the sample. Severe dysfunctions in eyes and ears age related changes were observed in 34 percent of urban and 33.5 percent of rural respondents. The common problems related to eyes were redness of eyes, pain, irritation and excessive watery eyes. Calculation of $\chi 2$ reveals that there was no significant difference found in the incidence of eyes and ears related problems between the two groups. 26 percent of males and 34 percent of females in urban areas and 17 percent of males and 28 percent females in rural areas were hard of hearing, also known as Presbycusis. Dimming eyesight and failing hearing can reduce physical, functional, emotional, and social well-being. Isolation, depression, and poorer social relationships often accompany sight and hearing loss. An awareness of different sensory changes and their effects can be helpful in improving quality of life.
\end{abstract}

Copyright (C) Kirti Sharma and Shubha Dube, 2017, this is an open-access article distributed under the terms of the Creative Commons Attribution License, which permits unrestricted use, distribution and reproduction in any medium, provided the original work is properly cited.

\section{INTRODUCTION}

The process of ageing begins at birth and continues throughout life. Sensory changes can influence the way we see, hear, taste, smell, and respond to touch and pain (Sell, 2014). This in turn affects how we experience the world and react to things. Sensory changes do not occur at the same age for each person, nor do all changes occur for everyone or to the same degree. The normal ageing process is accompanied by gradual decline in the various sensory modalities of audition, vision, gustation, olfaction, touch, proprioception, etc. A significant sensory change can rob us of many simple pleasures and complicate the tasks of daily living. It may mean reduced mobility, increased dependence on others, inaccurate perception of the environment, reduced ability to communicate and socialize, or loss of self-esteem (Tanner, 2006).

All these senses may be affected by ageing, though the dysfunctions of sensations of audition and vision are affected most. Changes in vision and hearing are particularly important because they can affect the person's ability to function in the physical environment and may lead to isolation. An awareness of different sensory changes and their effects can be helpful in improving quality of life (Sell, 2014).

Our eyes and our ears are how we make sense of the world around us. As people age, more of their balance abilities depend on vision. Thus with increasing visual impairment increased likelihood of falling. Even minor degrees of cognitive difficulty dramatically increase the impact of visual and hearing impairments. Simple tasks, such as injecting insulin, or taking the right pill at the proper time become very challenging with sensory impairment. Hearing-impaired ageing adults do not hear our instructions properly and often are too embarrassed to ask us to repeat, or do not even realize they have missed important information (Meiner, 2015).

For the present study the sample comprised of $\mathrm{N}=400$ ageing adults both males and females, in the age range of 65 to $75 y e a r s$ belonging to rural and urban setting of Jaipur district ( $\mathrm{n}$ $=200$ urban, i.e $100=$ male, $100=$ female and $n=200$ rural i.e. $100=$ male and $100=$ female). A selected question from Cornell Medical Index Health Questionnaire (Wig et al, 1999) was used to assess ear and eye related problems. A yes response answer indicates an individual's currently or previously 
occurring symptoms. An individual's C.M.I score is determining by the sum total of yes responses over the entire questionnaire. Depending upon total score, the respondents were categorized into high, medium and low level of each type of distress. The collected data was tabulated and analyzed using the statistical package SPSS-16.

\section{Eyes and Ears Problems in the Respondents}

All of the senses are important to good health and well being; vision and ear are the most important. The normal ageing process causes gradual losses to the sensory system. Significant losses to eyesight can reduce quality of life and threaten ability to live independently at home and in the community. Thus, it is important to understand how age affects the eye and ear, recognize the most common eye and ear problems, and learn ways to deal with poor eyesight and hard of hearing.

Table 1 Stages of severity of Eyes and Ears Age Changes

\begin{tabular}{|c|c|c|c|c|c|c|c|}
\hline \multicolumn{8}{|c|}{$(\mathrm{N}=400)$} \\
\hline \multirow{2}{*}{$\begin{array}{l}\text { Stage of } \\
\text { Severity }\end{array}$} & \multicolumn{2}{|c|}{ Urban $n=200$} & \multirow[b]{2}{*}{$\begin{array}{c}\text { Total } \\
\%\end{array}$} & \multicolumn{2}{|c|}{ Rural $n=200$} & \multirow[b]{2}{*}{$\begin{array}{c}\text { Total } \\
\%\end{array}$} & \multirow{2}{*}{$\begin{array}{c}\text { Chi Sq } \\
\text { (p-value) }\end{array}$} \\
\hline & $\begin{array}{c}\text { Male } \\
\%\end{array}$ & $\begin{array}{c}\text { Female } \\
\%\end{array}$ & & $\begin{array}{c}\text { Male } \\
\%\end{array}$ & $\begin{array}{c}\text { Female } \\
\%\end{array}$ & & \\
\hline Lov & 45 & 42 & 43.5 & 54 & 53 & 53.5 & \\
\hline Moderate & 19 & 19 & 19 & 12 & 14 & 13 & NS \\
\hline Severe & 36 & 39 & 37.5 & 34 & 33 & 33.5 & \\
\hline $\begin{array}{c}\text { Chi Square } \\
\text { (M vs F) }\end{array}$ & \multicolumn{3}{|c|}{$0.223(0.894) \mathrm{NS}$} & \multicolumn{3}{|c|}{$0.178(0.915) \mathrm{NS}$} & \\
\hline
\end{tabular}

Note. ${ }^{*} \mathrm{p}<.01 ;{ }^{*} \mathrm{p}<.05 ; \mathrm{NS}=$ Not Significant

Table 1 reveals that majority of the respondents i.e. 43.5 percent in urban and 53percent in rural areas had less (low) severe age - related charges associated with the sensations of audition and vision. This indicates that this population was satisfied with the condition of their eyes and ears. Severe dysfunctions in eyes and ears age related changes were observed in 34 percent of urban and 33.5 percent of rural respondents. The remaining population had moderately severe age - related changes in eye and ear. The common problems related to eyes were redness of eyes, pain, irritation and excessive watery eyes. Calculation of $\chi 2$ reveals that there was no significant difference found in the incidence of eyes and ears related problems between the two groups. Sensory changes can affect the lifestyle of a person and may undermine various day - to - day activities like communicating, enjoying activities, and staying involved with people; these changes can result in the phenomenon of isolation (Caprio, 2007).

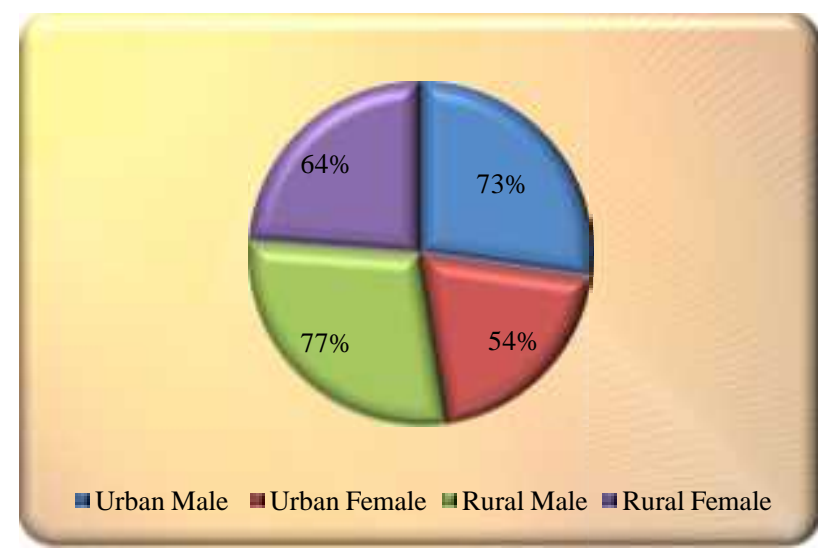

Figure 1 Presbyopia among ageing adults $(\mathrm{N}=400)$.
Figure 1displays specific eye problems among urban and rural ageing males and females. The figure indicates that 73 percent male and 54 percent female participants had problem in reading. Among rural sample 77 percent males and 64 percent females had reading difficulty. The structure of eye is change with aging. The pupils decrease to about one third of the size and react more slowly in response to darkness or bright light, lens becomes yellowed, less flexible and slightly cloudy, eye muscles become less able to fully rotate the eye, sharpness of vision (visual acuity) gradually declines, fat pads supporting the eyes decrease in amount and the eyes sink into their sockets. The most common problem is difficulty in focusing the eyes on something close. This condition is called Presbyopia (Minaker, 2011). A chi-square test of independence was performed to examine the difference between urban and rural, male and female respondents and reading problems. The relation between these variables was found to be significant at, $(\chi 2=7.778, p<0.05)$ in urban male and female respondents.

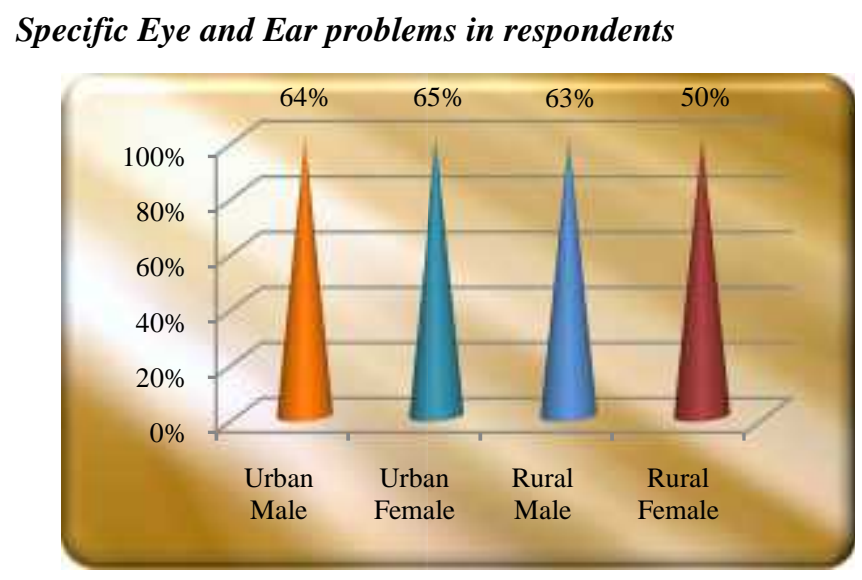

Figure 2 Myopia among ageing adults $(\mathrm{N}=400)$

Figure 2 clarifies that specific problem with distance vision among urban and rural respondents. It was observed from the figure that 64 percent urban males, 65 percent urban females and 63 percent rural males and 50 percent rural females had problem in distance vision. Myopia is the technical name for short - sightedness - a defect in vision that comes about when the eyes can't focus light from distant objects correctly; one can focus on close objects clearly, but distant objects are blurred. High myopia is a major cause of blindness and vision impairment.

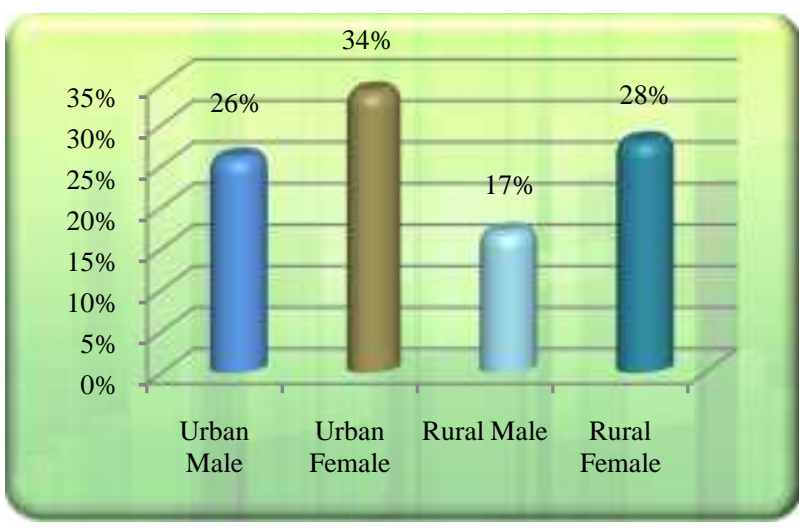

Figure 3 Presbycusis among ageing adults $(\mathrm{N}=400)$. 
The prevalence of retinal pathology increases with increasing age, axial length and refractive error (Chen, 2014; Seshamani, 2010). A very high Myopic condition was prevalent in both the section of the sample.

It is evident from the Figure 3 that 26 percent of males and 34 percent of females in urban areas and 17 percent of males and 28 percent females in rural areas were hard of hearing, also known as Presbycusis. Presbycusis (age-related hearing loss) is the loss of hearing that gradually occurs in most individuals as they grow older. It affects both ears. Hearing loss is a common disorder associated with ageing and is ranked as the third most prevalent chronic condition in elderly people after hypertension and arthritis. Hearing may decline especially for high frequency sounds, may have trouble telling apart certain sounds and may have problems in understanding others when there is background noise (Hile, 2007). Ruben, (2007) reported that between 30 to 40 percent of people aged 65 years and above have significant hearing loss. In some people structure of the ear become less elastic and undergo changes that make them less able to respond to sound waves, contributing to their hearing loss.

\section{Recommendations}

- Early diagnosis and treatment with medication can help prevent sensory loss.

- Avoid smoking and excessive caffeine consumption.

- Maintaining proper function depends on eating a balanced diet.

- Regular exercise stimulated good circulation and oxygen intake.

- Regular eye test to wear the proper prescription lenses. This will improve quality of life and reduce the risk of accidents such as falls.

- Good circulation and oxygen intake are important for our eye health. Both of these are stimulated by regular exercise.

- As people aged bright light can be used in homes for their better vision.

- Increase the daylight in by keeping windows clean and curtains pulled back. Good electric lighting, especially at the top and bottom of stairs.

- Sleep well, so the eyes are continuously lubricated and irritants, such as dust or smoke that may have accumulated during the day are cleared out.

- Use facial expressions, gestures, and visual aids to illustrate message. Write important information down as well as give it orally.

- Uses of special equipment such as hearing aids, walking sticks are useful.

\section{CONCLUSION}

Dimming eyesight and failing hearing can reduce physical, functional, emotional, and social well-being.
Visual and hearing impairments decrease independence in performing the activities of daily living, getting from place to place, or communicating with others.

Isolation, depression, and poorer social relationships often accompany sight and hearing loss. Visual and hearing impairments are directly related to quality of life. An increase in medical evaluations, early diagnosis and treatment with medication and the use of special equipment could greatly improve the quality of life for older people and decrease the level of disability associated with these impairments.

\section{Acknowledge}

Sponsored by UGC, New Delhi, Major research Project, entitled "An Intervention Study on Morbidity Pattern among Urban and Rural Ageing Adults"

\section{Reference}

Burton M \& Ludwig L. (2015). Fundamentals of nursing care: concepts, connections \& skills. Philadelphia: F. A. Davis.

Caprio, T., \& Williams, T.F. (2007). Comprehensive geriatric assessment. In: Duthie EH, Katz PR, Malone ML, eds. Practice of Geriatrics. 4th ed. Philadelphia, PA: Elsevier Saunders, chap 4.

Chen, 1., \& Brien, A. (2014). Retinal pathology in highly myopic eyes: ZOC-BHVI High Myopia Study, Brien Holden Vision Institute, Sydney, ACT, Australia, ARVO, Annual Meeting Abstracts, Zhongshan Ophthalmic Center, Guangzhou, China.

Hile, E.S., Studenski, S.A. (2007). Instability and falls. In: eds. Practice of Geriatrics.4th ed. Philadelphia, PA: Elsevier Saunders; chap 17.

Meiner S. (2015). Gerontologic nursing. Maryland Heights, MO: Elsevier Mosby, Inc.

Minaker, K.L. (2011). Common Clinical sequelae of aging. In: Goldman L, Schafer AI, eds. Goldman's Cecil Medicine. 24th ed. Philadelphia, PA: Elsevier Saunders, chap 24.

Ruben, M. D., \& Robert, J. (2007). Hearing Loss and Deafness. The Merck Manuals on Line Medical Library (Home Edition).

Sell C. (2014). Chemistry and the sense of smell. Hoboken, NJ: John Wiley \& Sons.

Seshamani, M., Kashima ML. Special considerations in managing geriatric patients. In: Flint PW, Haughey $\mathrm{BH}$, Lund LJ, et al, eds. Cummings Otolaryngology: Head \& Neck Surgery. 5th ed. Philadelphia, PA: Elsevier Mosby, chap 16.

Tanner D. (2006). Medical-legal and forensic aspects of communication disorders, voice prints and speaker profiling. Tucson: Lawyers and Judges Publishing Company.

Wig, N.N, Pershad, D, \& Verma, S.K., (1999). Manual for C.M.I. Health questionnaire. National psychological corporation.

\section{How to cite this article:}

Kirti Sharma and Shubha Dube.2017, Audition and Vision Changes among Geriatric Residents. Int J Recent Sci Res. 8(3), pp. 16027-16029. 